\title{
Der Oasis Park auf Fuerteventura, ein Vergnügungspark mit botanischem Garten
}

\author{
Stephan Scholz \& Hans Grasmück
}

\begin{abstract}
Zusammenfassung
Der Oasis Park auf der Kanareninsel Fuerteventura ist ein großer Freizeitpark, in dem ein Zoo und ein botanischer Garten integriert sind. Letzterer wird hier vorgestellt. In ihm werden kanarische Arten inklusive seltener Endemiten kultiviert. Pflanzen der verschiedenen Ökosysteme der Kanaren wie Sukkulentenbusch und thermophiler Wald werden vorgestellt. Der Garten beherbergt auch zahlreiche andere interessante nicht-kanarische Pflanzen, unter ihnen tropische Baum- und Straucharten, viele verschiedene Palmen sowie eine große Sukkulenten-Sammlung mit Kakteen, Agaven und Aloe-Arten, Sukkulenten aus Madagaskar und Yuccas.
\end{abstract}

\begin{abstract}
Oasis Park Fuerteventura is a big amusement park with a zoological and a botanical garden. The latter is described here. In this garden, plants of the Canary Islands are cultivated, including rare endemic species. Plants of different ecosystems like succulent scrub and thermophilous forest are displayed. The garden also hosts many other plants, including tropical tree and shrub species and many different palms, as well as a large succulent plant assemblage with cacti, succulent plants from Madagascar, aloes, yuccas and agaves.
\end{abstract}

\section{Einleitung}

In der Nähe des Fischerdorfes La Lajita, im Südosten von Fuerteventura, befindet sich der Oasis Park, eine interessante Mischung aus Vergnügungspark, Zoo und botanischem Garten. Die Vielfalt an Arten, aber auch die Historie dieser bemerkenswerten Anlage, haben uns motiviert, über die interessanten botanischen Aspekte zu berichten.

Gegründet wurde der Park 1988 von zwei Familien aus Fuerteventura, die sich bis dahin der Landwirtschaft und Viehzucht gewidmet hatten. Die Idee war, diese traditionellen Aktivitäten im Hinblick auf den wachsenden Fremdenverkehr zu nutzen. Nach der Anfangsphase mit zwei Dromedaren, auf denen Besuchergruppen Ausflüge zum nahegelegenen Strand machten, kamen Gärtnerei, zoologischer und botanischer Garten dazu, die ständig erweitert wurden. Heute empfängt der Oasis Park ca. 300000 Besucher im Jahr, beschäftigt über 200 Personen und ist der größte Freizeitpark der Kanaren.

\section{Natürliche Gegebenheiten und Anbaubedingungen}

Der Park liegt zwischen 30 und 200 m Höhe in felsigem, nach Südosten zur Küste abfallendem
Terrain. Die mittlere Jahrestemperatur beträgt $20^{\circ} \mathrm{C}$, die mittlere Niederschlagsmenge liegt bei $100 \mathrm{~mm}$. Unterhalb einer halbmeterhohen, lehmigen, stark kalk- und etwas salzhaltigen Bodenschicht steht hartes, unverwittertes, aber teilweise stark zerklüftetes Basaltgestein an, das mit Schichten aus vulkanischer Asche und Agglomeraten abwechselt. Wegen der geringen Niederschlagswerte werden alle Pflanzen einschließlich der Sukkulenten mit Tropfbewässerung versorgt. Benutzt wird entsalztes Meerwasser, das teils in einer eigenen Anlage produziert, teils zugekauft wird. Es kommt auch geklärtes Abwasser zur Anwendung. Das Wasser kann in Gesteinsspalten verhältnismäßig tief eindringen, wodurch das Wachstum der Pflanzen begünstigt wird. In manchen Teilen der Anlage ist der Boden zusätzlich mit einer Schicht aus Vulkanasche abgedeckt, was die Verdunstung herabsetzt.

\section{Oberes Gartenareal: Kanarenflora}

Dieses 6 ha große Gebiet dient der Kultivierung und Untersuchung der einheimischen Flora Fuerteventuras und Lanzarotes, der beiden Ostinseln der Kanaren. Der Oasis Park arbeitet mit den Umweltbehörden der Inselverwaltung zusammen und hat ein Kooperationsabkommen 


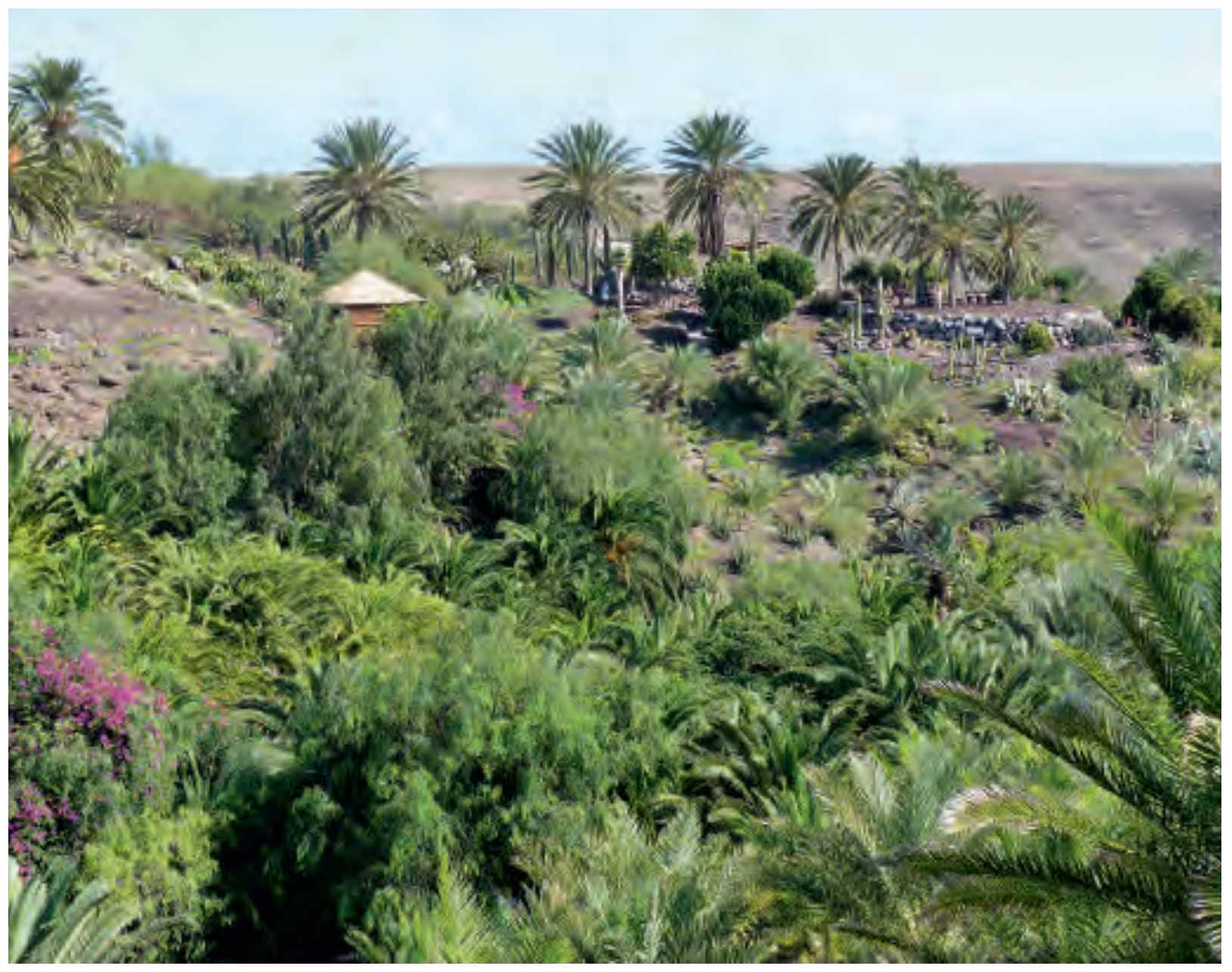

mit dem Botanischen Garten „Viera y Clavijo“ auf Gran Canaria. Vorrangige Aufgabe ist die Erhaltung gefährdeter endemischer Pflanzen. Umwelterziehung, z. B. durch geführte Besuche für Schulklassen, ist ebenfalls ein wichtiges Arbeitsgebiet. In diesem Areal sind außerdem Teiche mit Ufervegetation aus Pfahlrohr und Rohrkolben untergebracht, wo Wasservögel wie die seltene Rostgans (Tadorna ferruginea) brüten und Zugvögel Schutz finden.

Von den 2091 wildwachsenden Gefäßpflanzenarten des Archipels, von denen 537 dort endemisch sind, kommen 732 Arten auf Fuerteventura und 686 auf Lanzarote vor (ACEBES et al. 2010). Als größte botanische Kostbarkeiten hat jede dieser beiden Inseln circa ein Dutzend Lokalendemiten (die Angaben variieren

Abb. 1: Allgemeine Ansicht des Gartens. aufgrund der unterschiedlichen taxonomischen Bewertung mancher Sippen). Fast alle sind stark durch die vor allem auf Fuerteventura verbreitete extensive Ziegenhaltung sowie durch Bautätigkeit und den Klimawandel bedroht. Ziel ist es, die Arten innerhalb ihrer jeweiligen Pflanzengesellschaften zu erhalten. Bei La Lajita ist wie in den meisten Teilen Fuerteventuras keine natürliche potenzielle Vegetation mehr vorhanden; vorherrschend sind nitrophile Ersatzgesellschaften mit Dornlattich (Launaea arborescens), Salsola- und Suaeda-Arten sowie dem Bocksdorn (Lycium intricatum). Dazu kommen in regenreichen Wintern Therophyten-Gesellschaften. Deshalb müssen die ursprünglichen Pflanzengemeinschaften durch die Anpflanzung größerer Individuenzahlen ihrer Komponenten nachgebildet werden. Dabei wird auch bei Arten, die ebenfalls auf anderen Inseln der Kanaren vorkommen und dort häufiger als auf Fuerteventura sind, darauf ge- 
achtet, dass Exemplare von Fuerteventura vermehrt und gepflanzt werden, das genetische Material also von der Insel stammt.

Den Schwerpunkt bilden zwei Ökosysteme: Sukkulentenbusch und thermophiler Wald. Ersterer besteht vorwiegend aus Vertretern der Gattung Euphorbia und der Komposite Kleinia neriifolia und bedeckt in verschiedenen Varianten die trockenen Küstengebiete besonders der Südseiten aller Inseln. Auf Fuerteventura ist er in über die Insel verteilten kleinen Resten vorhanden. Der thermophile Wald kommt in niederschlagsreicheren, aber ebenfalls sommertrockenen Berggebieten vor. Auf Fuerteventura bestand er vorwiegend aus wilden kanarischen Ölbäumen (Olea cerasiformis) und Pistacia atlantica. Die verbliebenen Reste sind so klein, dass man nicht mehr von Wald sprechen kann. In den Steilwänden des Jandía-Gebirges, das mit dem 807 m hohen Pico de la Zarza und dem nur wenig niedrigeren Pico del Mocán die höchsten Erhebungen der Insel besitzt, die im
Sommer von der Passatwolke befeuchtet werden, haben sich auch wenige Hektar einer endemitenreichen, feuchten Variante des termophilen Waldes gehalten. Es handelt sich dabei um eine Art Nebelwald, den man auf Fuerteventura nicht erwartet und der in Physionomie und Artenzusammensetzung den Lorbeerwaldgesellschaften der Westinseln nahekommt (Martín et al. 2011).

Im Garten wird versucht, den unterschiedlichen Ansprüchen dieser Lebensgemeinschaften dadurch gerecht zu werden, dass die Vertreter des Sukkulentenbusches auf exponierten Hängen mit starker Sonneneinstrahlung und die Vertreter des thermophilen Waldes in nach Norden ausgerichteten, schattigeren kleinen Schluchten und Rinnen angesiedelt wurden. Es ist nicht möglich, die feucht-kühlen Bedingungen des oberen Jandía-Gebirges im Garten bereit zu stellen, sodass die Pflanzengesellschaften des Nebelwaldes nur unvollständig nachgebildet werden können. Kleinere Flächen zeigen

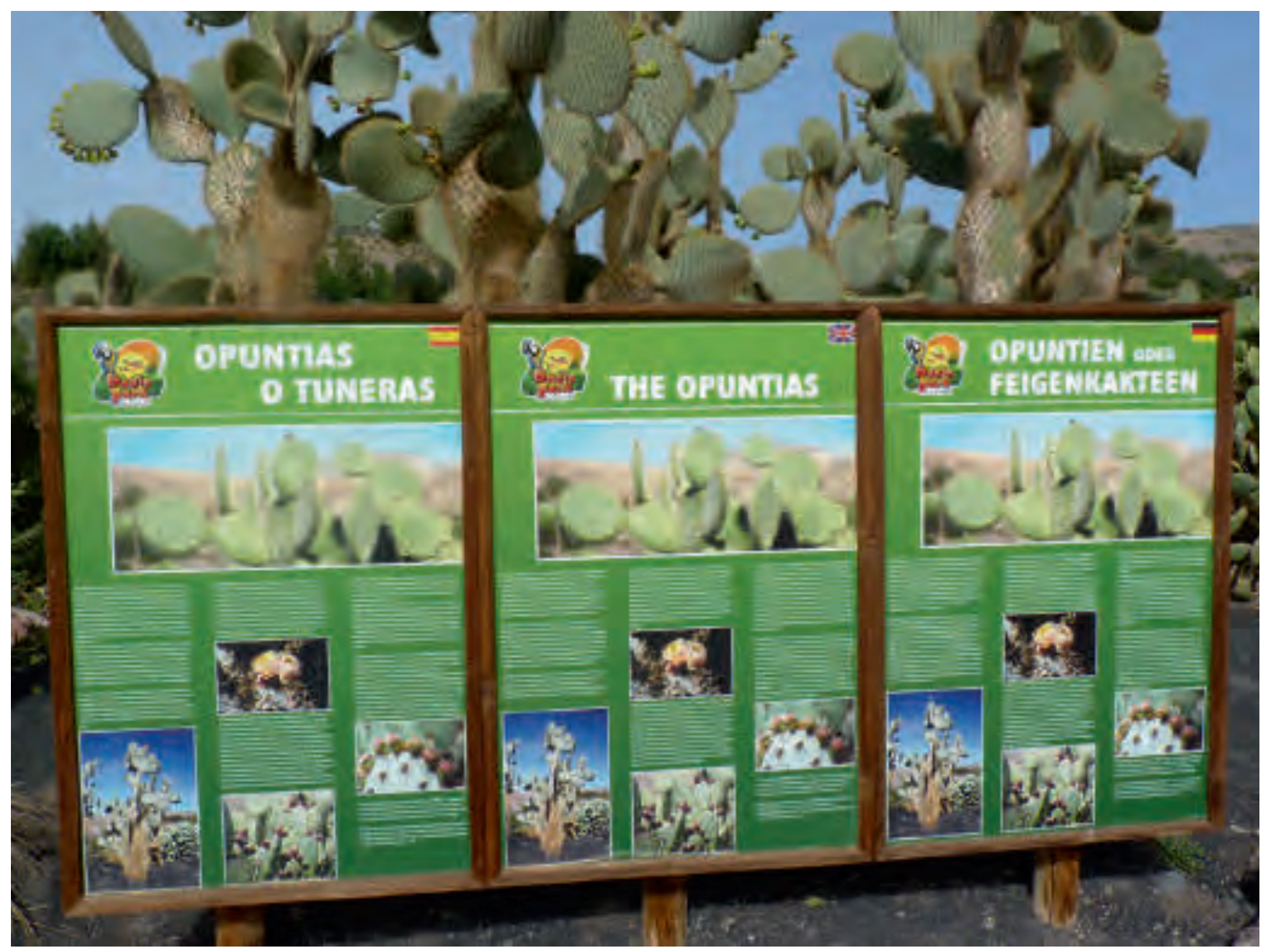


zusätzlich Vertreter der salztoleranten Sandküsten-Vegetation.

In den letzten drei Jahren wurden insgesamt über $1800 \mathrm{im}$ Garten selbst und in einer Gärtnerei der Inselverwaltung gezogene junge Euphorbia canariensis, Euphorbia balsamifera, Euphorbia regis-jubae und Kleinia neriifolia (= Senecio kleinia) gepflanzt. Letztere ist eine auch in Europa manchmal angebotene, kandelaberähnlich wachsende Art, auch Oleanderblättrige Kleinie oder Affenpalme genannt. Der Kanarenendemit ist zusammen mit den Euphorbien namengebend für die Vegetationsklasse Kleinio-Euphorbietea, in der die Pflanzengesellschaften des kanarischen Sukkulentenbusches zusammengefasst werden. Diese Pflanzungen bilden den Grundstock des Ökosystems Sukkulentenbusch, in das nach und nach etwa 40 andere Arten in unterschiedlicher Individuenzahl integriert werden. Eine Auswahl davon wird nachfolgend beschrieben.

Dreizehn endemische, strauchige Arten der Gattung Crambe sind bisher von den Kanaren beschrieben worden. Crambe sventenii ist die einzige auf Fuerteventura. Sie wurde nach dem schwedischen Erforscher der Kanarenflora, ERIK R. Sventenius (1910-1973), benannt und kommt sehr selten an unzugänglichen Felsen im Südosten der Insel vor. Gymnosporia cryptopetala ist ein dorniger, zweihäusiger Strauch, von dem auf Fuerteventura und Lanzarote insgesamt nur 15 Exemplare bekannt sind. Er wurde jahrzehntelang für den variablen, von Südspanien bis nach Südafrika verbreiteten Maytenus senegalensis gehalten, bis er 2010 von zwei kanarischen Botanikern als eigene, in wesentlichen Merkmalen von $M$. senegalensis abweichende Art erkannt und beschrieben wurde (REyesBetancort \& Santos 2010). Im botanischen

Abb. 2 (Seite 52): Beispiel für die im Garten aufgestellten Informationstafeln.

Abb. 3 (oben): Crambe sventenii.

Abb. 4 (Mitte): Ferula arnoldiana.

Abb. 5 (unten): Gut erhaltener Restbestand von Euphorbia canariensis.
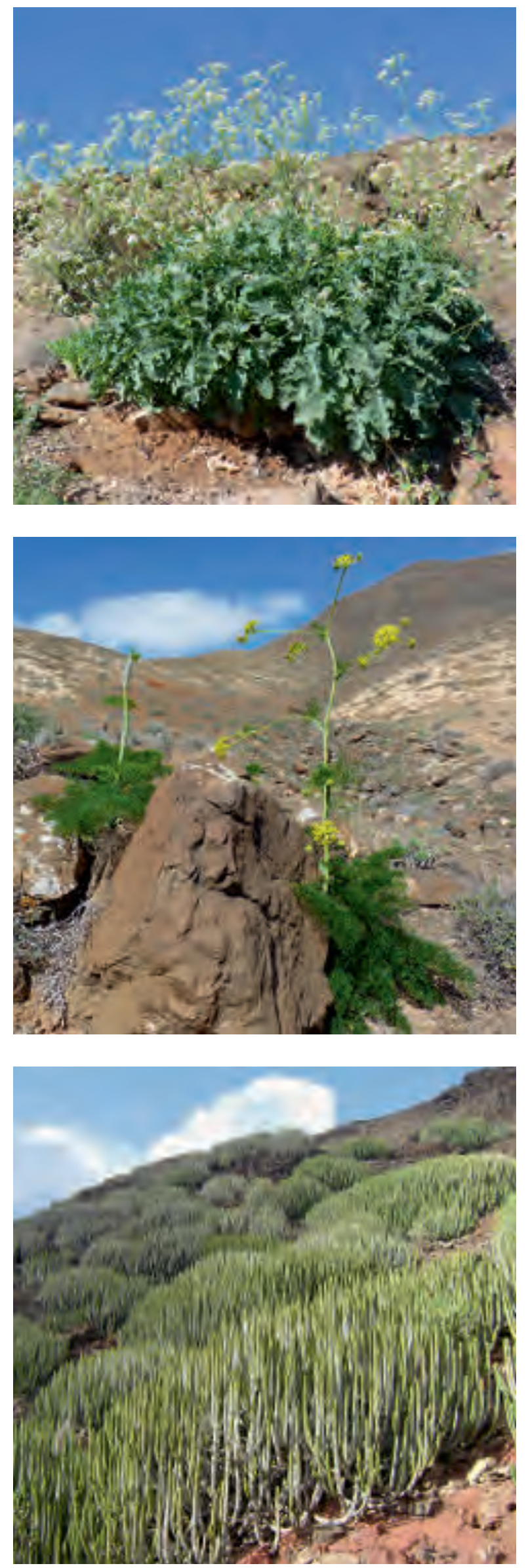

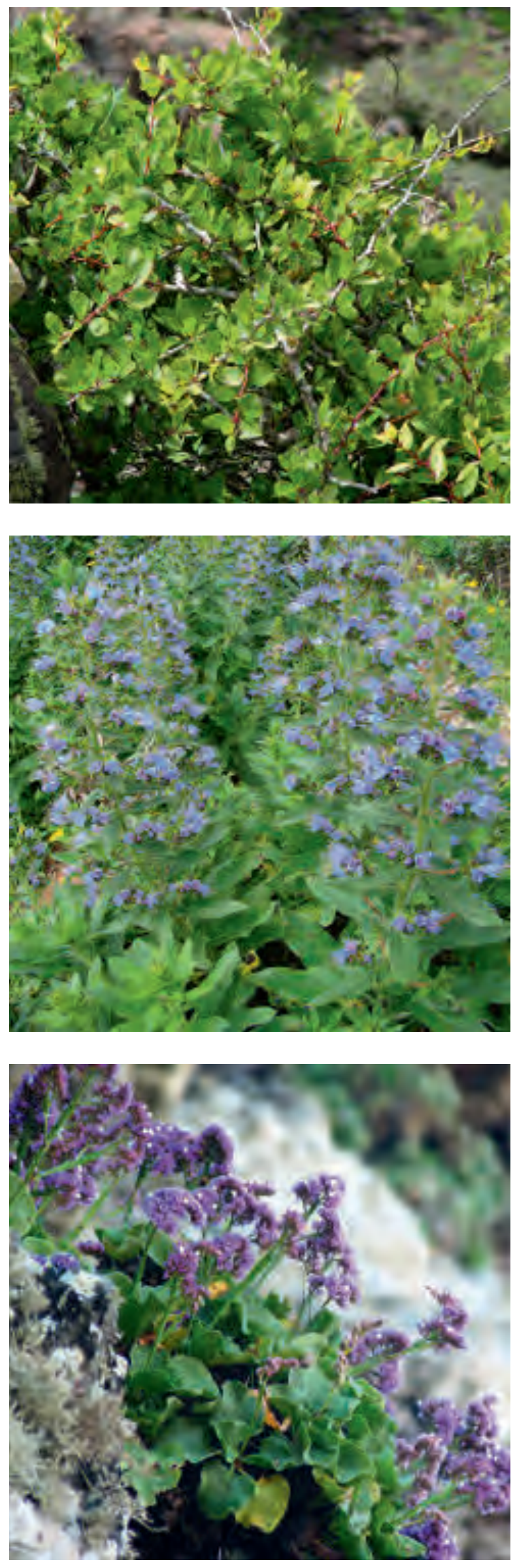

Garten sind 12 aus Samen und Stecklingen vermehrte Exemplare gepflanzt und werden weiter vermehrt. Bourgeaus Strandflieder (Limonium bourgeaui) ist wie die anderen 15 endemischen strauchigen Strandflieder-Arten der Kanaren eine schöne Zierpflanze. Sie ist im FamaraGebirge im Norden Lanzarotes stellenweise leicht zu finden. Vor 30 Jahren wurde sie auch im $150 \mathrm{~km}$ weiter südlich gelegenen JandíaGebirge auf Fuerteventura entdeckt (SANTOS \& Fernández 1984), das viele floristische Gemeinsamkeiten mit Famara aufweist. Mit kaum 80 bekannten Exemplaren ist L. bourgeaui in Jandía stark gefährdet; die Vermehrung über Samen ist aber nicht schwer und die Anzahl der im botanischen Garten kultivierten Exemplare nimmt ständig zu. Der Fuerteventura-Salbei (Salvia herbanica) ist ein kleiner, einem Rosmarin ähnlicher Felsenstrauch aus den Bergen im Südosten Fuerteventuras, der mit $S$. chudaei aus den Gebirgen der Zentral-Sahara verwandt ist (Santos \& Fernandez 1986) und nach molekulargenetischen Untersuchungen (WILL \& Classen-Bockhoff 2014) auch mit der in den nordafrikanischen Trockengebieten weit verbreiteten $S$. aegyptiaca, mit den südarabischen $S$. areysiana und $S$. geminata sowie mit $S$. bariensis aus Somalia. Die um 1990 bekannte Anzahl von ca. 600 Individuen hat nach unseren eigenen Beobachtungen seitdem ständig abgenommen. Weniger als 50 Exemplare wachsen an unzugänglichen Felsen, sind daher vor Ziegen und Kaninchen geschützt und können fruchten. Einige alte, auf 60-70 Jahre geschätzte Exemplare sind uns seit 25 Jahren bekannt und haben sich seitdem kaum verändert.

Weitere Sukkulentenbusch-Arten der Anlage sind Asparagus arborescens, A. nesiotes subsp. purpuriensis, Caralluma burchardii, Campylan-

Abb. 6 (oben): Gymnosporia cryptopetala am Naturstandort.

Abb. 7 (Mitte): Echium handiense.

Abb. 8 (unten): Limonium bourgeaui am Naturstandort in den Jandía-Bergen.

Abb. 9 (Seite 55): Feuchtzone im oberen Jandía-Gebirge. 
thus salsoloides, Ceballosia fruticosa, Convolvulus floridus, Lavandula canariensis, Periploca laevigata, Plocama pendula, Rubia fruticosa und Sonchus pinnatifidus. Caralluma burchardii (Burchards Fliegenblume) ist eine bis $15 \mathrm{~cm}$ hohe Giftpflanze der Ostkanaren und Marokkos. Sie wächst rasenbildend und treibt sukkulente, kaum verzweigte, grüne bis graue vierkantige Sprosse, die an den Kanten nach unten gerichtete Zähnchen tragen. Die Blüten haben fünf dreieckige, innen purpurbraune, weiß behaarte Zipfel. Sie bildet $10 \mathrm{~cm}$ lange, violett gesprenkelte, in Paaren hornförmig stehende Balgfrüchte, in denen sich zahlreiche flache, braune, mit einem Haarbüschel versehene Samen befinden. Auch die endemische JandíaWolfsmilch (Euphorbia handiensis) wird im botanischen Garten kultiviert. Auf anderen Inseln der Kanaren, aber nicht auf Fuerteventura einheimische Pflanzen wie Ceropegia fusca (Rotbraune Leuchterblume) und Schizogyne sericea könnten in einer späteren Phase dazukommen.
Die meisten Arten des thermophilen Waldes sind auf Fuerteventura viel seltener und wachsen an unzugänglicheren Stellen als die des Sukkulentenbuschs. Außerdem fruchten die wenigen verbliebenen Exemplare einiger Arten aus verschiedenen Gründen nicht. Einen repräsentativen Grundstock an Pflanzen für den botanischen Garten zu bekommen war nicht leicht. Bei extrem seltenen Arten wie Sideroxylon canariense, von dem nur ein einziges Exemplar auf Fuerteventura bekannt ist, gelang die Vermehrung durch vegetative Techniken erst nach Jahren. Arnoldos Riesenfenchel (Ferula arnoldiana) ist eine kürzlich beschriebene endemische Art aus dem Jandía-Gebirge (Scholz \& Reyes-Betancort 2013), dessen Gesamtbestand auf etwa 100 wildwachsende Exemplare geschätzt wird. Wie bei den drei anderen kanarischen Ferula-Arten sterben die oberirdischen Teile im Sommer ab. Im Herbst treibt der fleischige Wurzelstock wieder aus; im Februar kommt die Pflanze zur Blüte. Von den 19 ende-

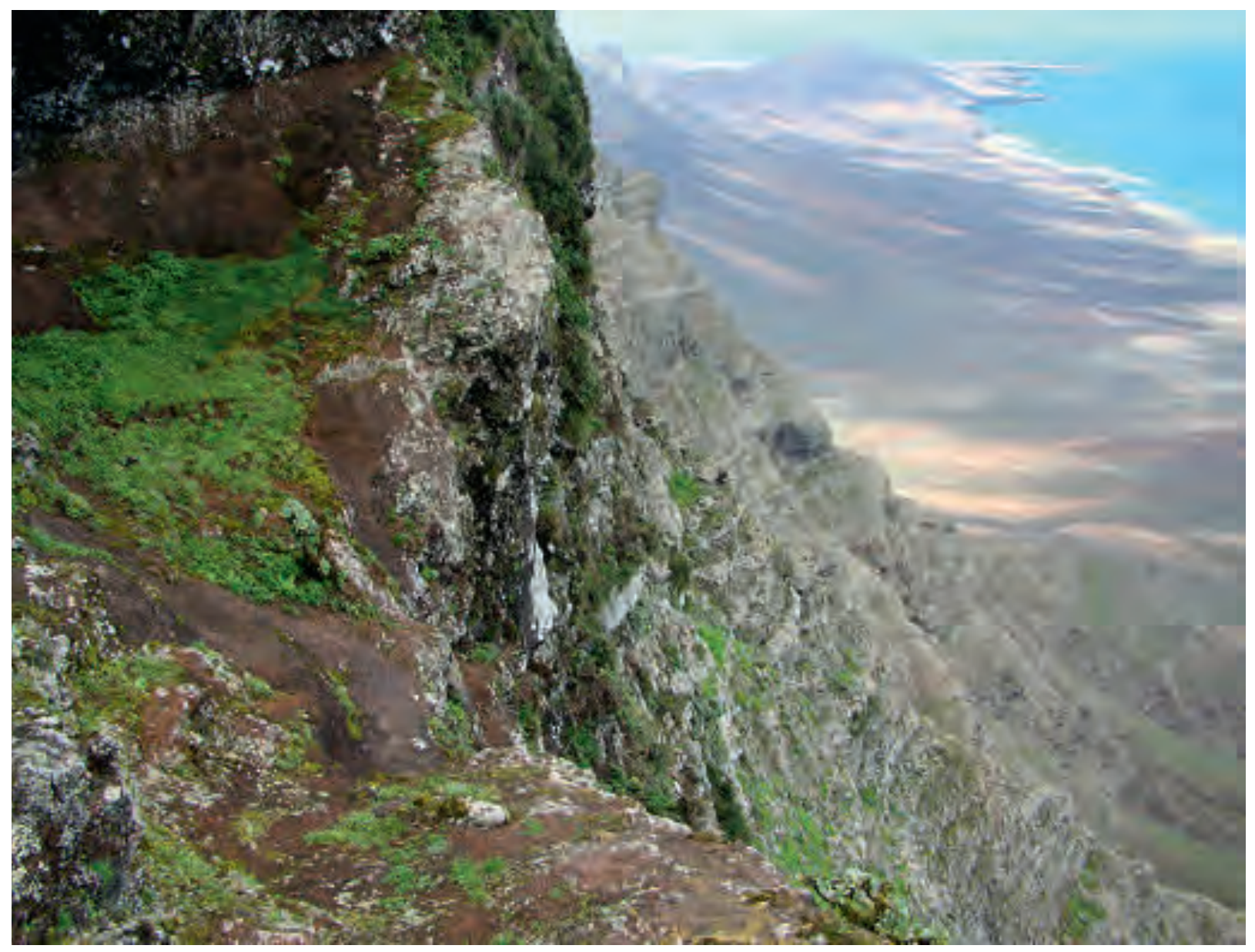



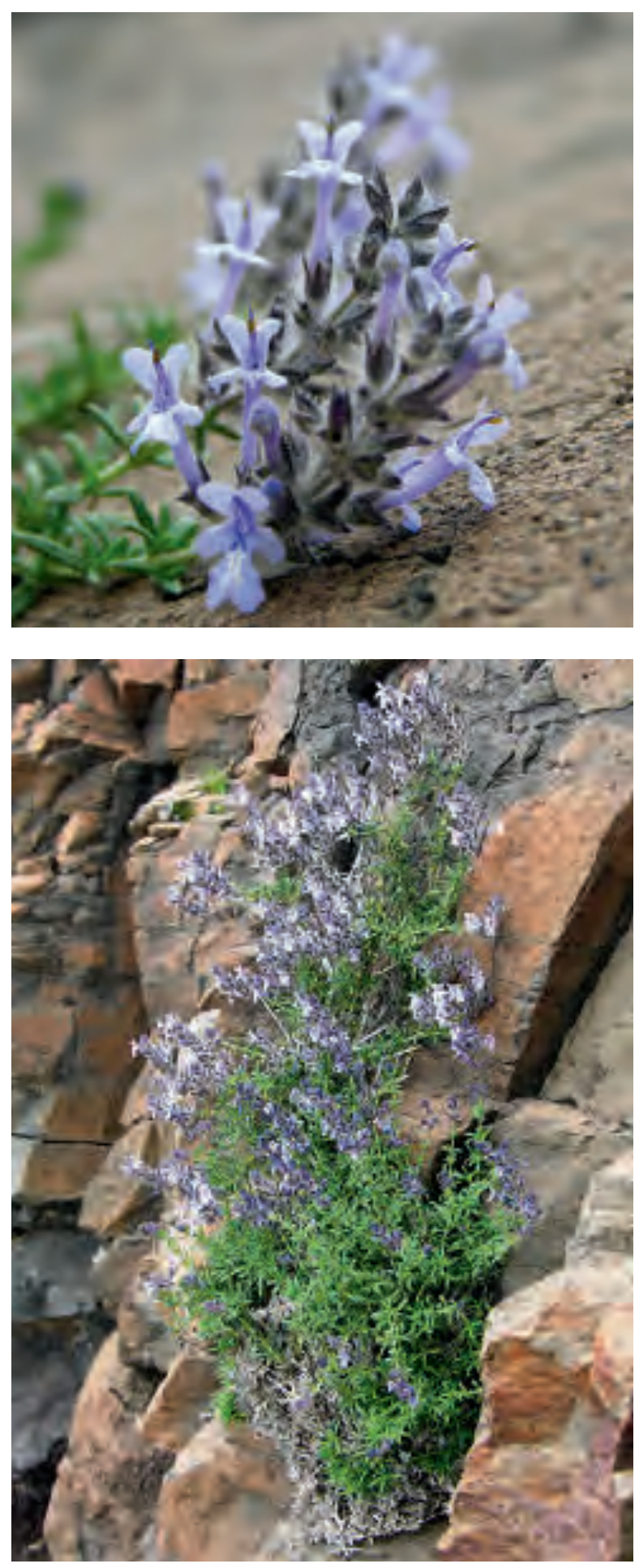

mischen Strauchmargariten der Kanaren ist Winters Strauchmargarite (Argyranthemum winteri) die einzige auf Fuerteventura. Vier kleine Relikt-Populationen der Art sind bekannt. Sie blüht von Februar bis April. Die Vermehrung gelingt außer über Samen auch aus Stecklingen. Der Jandía-Natternkopf (Echium handiense) ist ein kleiner Strauch, der nur in den höchsten Lagen des Jandía-Gebirges vorkommt. Eine schöne Zierpflanze, die aber relativ feuchte und kühle Bedingungen braucht. Der Gekerbtblättrige Kreuzdorn (Rhamnus crenulata) wächst zu einem ausladenden Strauch aus. Auf Fuerteventura sind ein paar Dutzend Exemplare bekannt. Die Vermehrung ist bisher nur über die dunklen Beerenfrüchte gelungen, die im Herbst reifen. Die Atlantische Pistazie (Pistacia atlantica) hat eine weite Verbreitung von den Kanaren über Nordafrika bis zum Iran und kommt auf Fuerteventura in einem eng begrenzten Gebiet im Betancuria-Massiv im Inselinneren vor. Der Baum ist im Winter laubabwerfend. Er hat kleine, kaum essbare Samen, kann aber als Pfropfunterlage für die Echte Pistazie (Pistacia vera) benutzt werden. Weitere im botanischen Garten zu sehende Pflanzen des thermophilen Waldes sind der Seidige Goldstern (Asteriscus sericeus), Bupleurum handiense, Bosea yervamora, Maytenus canariensis, Scilla iridifolia und Visnea mocanera. Der jährliche Vegetations- und Blühzyklus des Schwertlilienblättrigen Blausterns (Scilla iridifolia) fängt im Herbst an. Bekommen die Pflanzen in der Natur im November und Dezember genügend Regen, stehen sie Weihnachten in voller Blüte und fruchten im März. Die Art ist im Gegensatz zu der auf Fuerteventura weit verbreiteten Meerzwiebel (Urginea maritima) sehr selten und meist an unzugänglichen Felsen zu finden. Ein Grund dafür könnte sein, dass ihre Zwiebeln, wie wir beobachtet haben, im Sommer von Kaninchen gefressen werden; sie haben möglicherweise einen geringeren Gehalt an herzwirksamen Glykosiden als die hochgiftige Meerzwiebel, die von Kaninchen und Ziegen nicht angerührt wird.

\section{Kakteen- und Sukkulentengarten}

Der größte Garten seiner Art auf den Kanarischen Inseln weist auf 4 ha Fläche einige Tau-

Abb. 10 (oben): Blühendes Exemplar von Salvia herbanica.

Abb. 11 (unten): Salvia herbanica an einem Felsen.

Abb. 12 (Seite 57): Eingang zum Sukkulenten-Garten. 
send Pflanzenindividuen in knapp 200 Arten auf. Er liegt auf einem nach Süden und Südosten geneigten Hang, der in einer Talsenke ausläuft. Die Wegführung ist dem hügeligen Gelände angepasst, sodass abgegrenzte Areale geschaffen wurden, die die separate Darstellung oder Gruppierung bestimmter Pflanzengruppen ermöglichen. Dieser Teil des Gartens soll vor allem die zahlreichen Feriengäste anziehen. Viele Arten, wie z. B. der Schwiegermutter-Sitz (Echinocactus grusonii), sind in großen Stückzahlen vorhanden und ergeben eindrucksvolle Fotomotive. Didaktisch wertvoll sind die Informationstafeln über ausgewählte Pflanzengruppen und die wissenschaftliche Ausschilderung mittels Etiketten. Kleine Pavillons, Bänke und Sitzecken sowie schön blühende Schattenbäume geben den Besuchern während des lehrreichen Rundganges durch die Sukkulentenanlage die Möglichkeit zum Ausruhen. Neben den Kakteen gibt es im Garten auch viele Agaven, darunter auch die attraktive Agave attenuata mit ihrem schwanenhalsähnlich gebogenen Blütenstand.

Von der Gattung Yucca werden rosettenbildende und baumförmige Arten kultiviert. Auf den Kanaren dürfen natürlich auch die Drachenbäume nicht fehlen. Dem makaronesischen Dracaena draco ähnelt in Größe und Gestalt am meisten $D$. cinnabari, der auf der Insel Sokotra endemisch ist. Der erst vor 15 Jahren im Süden von Gran Canaria entdeckte, extrem seltene Dracaena tamaranae ist dagegen der südarabischen Art D. serrulata am ähnlichsten. Die in der Anlage zahlreichen makaronesischen Drachenbäume mit ihren vorzeitlich anmutenden grauen Stämmen (Stammsukkulenz) zeigen im Jugendstadium noch keine Verzweigungen. Sie blühen zum ersten Mal nach 8 bis 25 Jahren, verlieren ihren endständigen Vegetationspunkt und stellen ihr Längenwachstum ein. Die nach der Blüte folgende Verzweigung bricht in vier bis acht schräg gestellten Sprossen wirtelig unter der alten Spitze hervor. Der an der Luft rot-

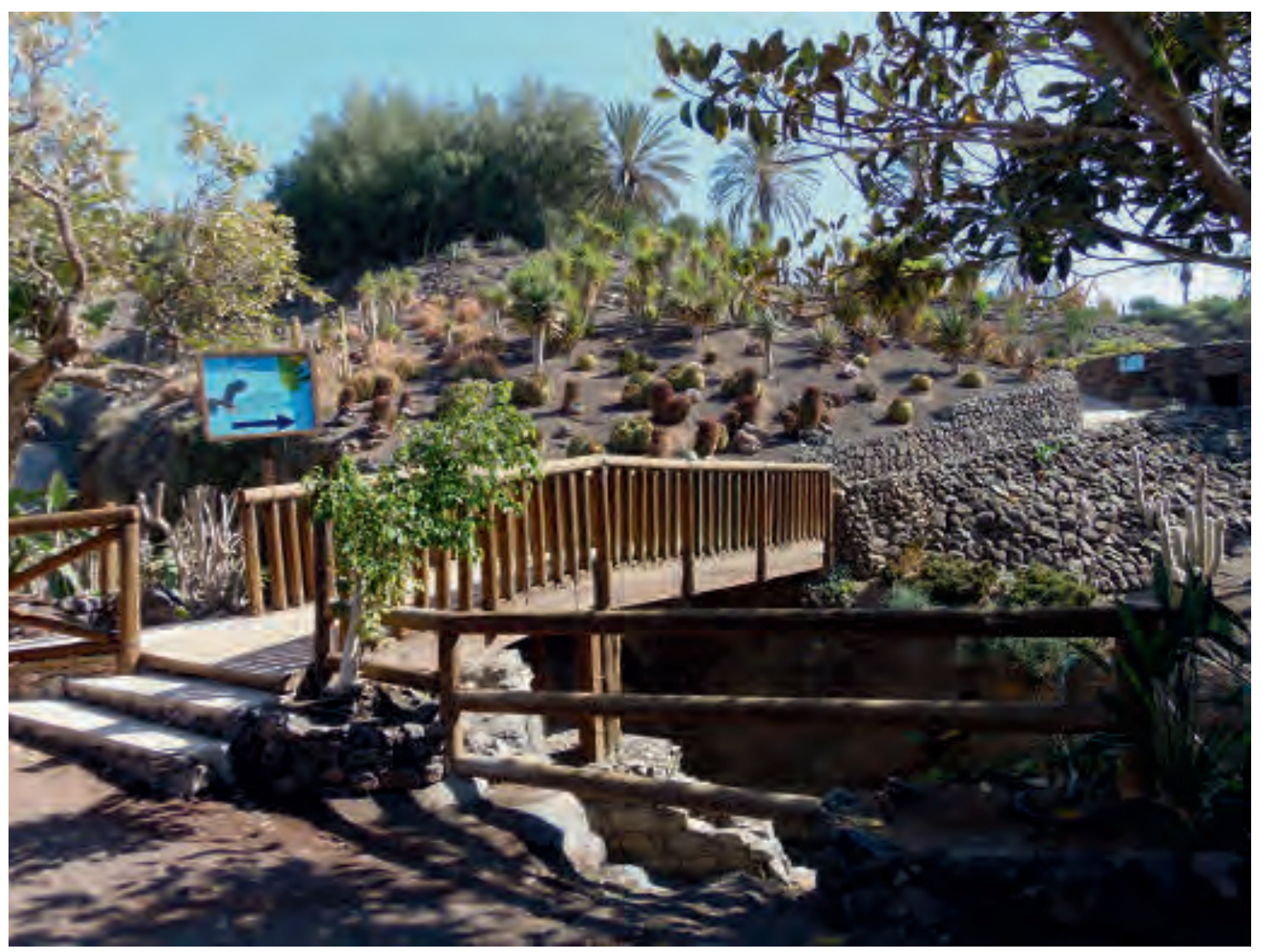



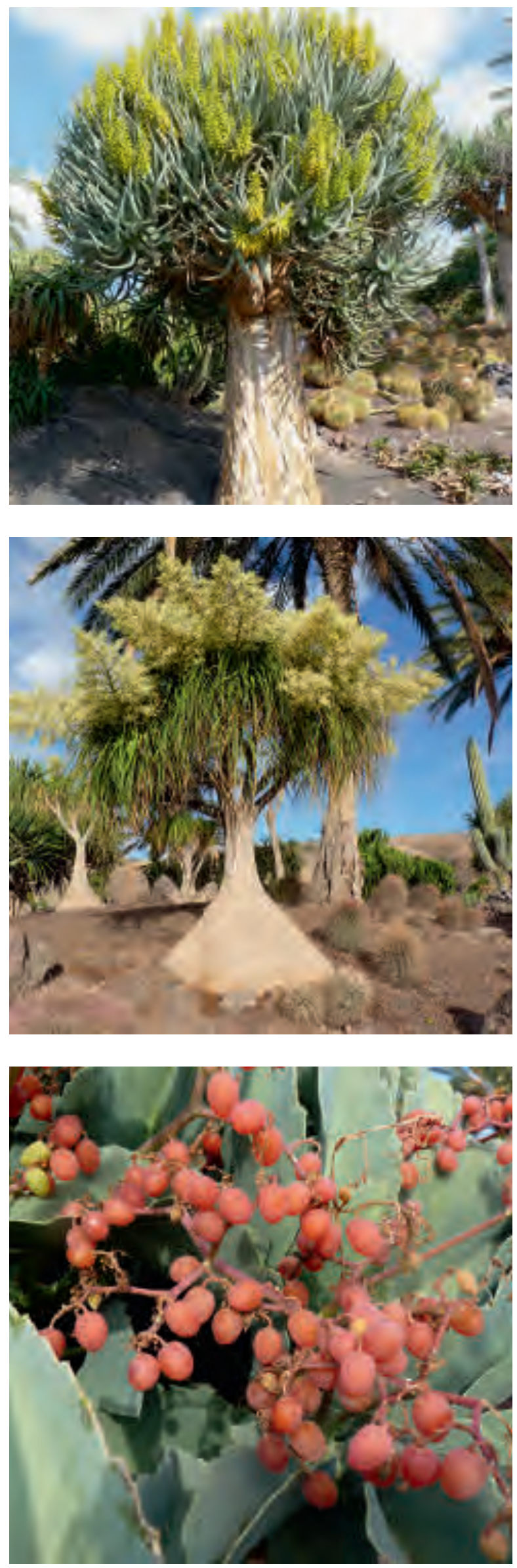

braun trocknende Saft des Drachenbaums hatte in der Heilkunde der Ureinwohner der Kanaren große Bedeutung und war im Mittelalter in Europa unter dem Namen Drachenblut als Heil- und Zaubermittel geschätzt. Mehrere gut entwickelte Elefantenfuß-Bäume (Beaucarnea recurvata $=$ Nolina recurvata) stehen ungefähr in der Mitte des Sukkulentengartens bei einer Aussichtsplattform, von der man einen schönen Blïck über den größten Teil der Anlage hat. Die Pflanze stammt aus den Trockengebieten Mittelmexikos, ist unregelmäßig verzweigt und kann bis zu 9 m hoch werden. Der verholzte Stamm trägt am Ende einen Schopf herabfallend gedrehter Blätter und ist in Bodennähe stark verdickt.

Von den im Garten vorhandenen 40 AloeArten ist eine Gruppe von Aloe dichotoma zu eindrucksvollen Exemplaren herangewachsen. Diese Aloe, in Südafrika Kokerboom genannt, weil die ausgehöhlten Äste von den Buschmännern als Köcher verwendet wurden, ist eine baumförmige, im Herbst intensiv gelb blühende Art und erreicht am Heimatstandort $8 \mathrm{~m}$ Höhe. In Gruppen im Garten platziert findet sich auch die in der Heilkunde beliebte Echte Aloe. Auf Fuerteventura gibt es große Plantagen dieser Art. Das Gel, das man aus dem wasserspeichernden Gewebe der Blätter von Aloe vera gewinnt und das entzündungshemmende, wundheilende und das Immunsystem stimulierende Eigenschaften besitzt, wird kosmetisch und volksmedizinisch in einer Reihe von Anwendungen eingesetzt. $\mathrm{Zu}$ sehen ist auch Aloe thraskii mit sehr langen, abwärts gerichteten Blättern und zitronengelben bis orange gefärbten Blüten sowie Aloe arborescens (Baum-Aloe), die eher strauchartig wächst und mehrere verzweigte Stämme entwickelt, die 2 bis $3 \mathrm{~m}$ hoch werden.

Abb. 13 (oben): Aloe dichotoma.

Abb. 14 (Mitte): Allgemeine Ansicht des SukkulentenGartens mit Beaucarnea recurvata.

Abb. 15 (unten): Die ungenießbaren Früchte von Cyphostemma juttae. 
In der Natur sind die Crassulaceae auf den Kanarischen Inseln besonders durch die Gattung Aeonium vertreten, von deren je nach Auffassung 35 bis über 40 Arten alle bis auf sechs auf den Kanaren endemisch sind. Im Garten sind u.a. Aeonium balsamiferum und A. lancerottense zu sehen. Beide sind nur auf Lanzarote einheimisch, auf Fuerteventura findet man A. balsamiferum aber verwildert, weil sein Saft früher zum Konservieren von Netzen und Fischer-Leinen aus Naturfasern benutzt wurde. Auch die Vitaceae umfassen sukkulente Vertreter. In Europa als Zimmerpflanze bekannt sind Arten der Gattung Cissus und seltener Cyphostemma. Im Sukkulentengarten des Oasis Parks befinden sich große Exemplare von $C y$ phostemma juttae (=Cissus juttae). Die Art stammt aus heißen und trockenen Gebieten Namibias und ist ein nicht kletterndes Weinrebengewächs, dessen weicher, Wasser speichernder Stamm in der Heimat 2 bis $3 \mathrm{~m}$ hoch und am Grunde bis zu $1 \mathrm{~m}$ dick werden kann. Im Garten sind zudem 25 Euphorbia-Arten angesiedelt. Auffällig sind besonders E. abyssinica, $E$. ingens und $E$. candelabrum. Letztere entwickeln sich zu massigen, bis $12 \mathrm{~m}$ hohen Kandelaber-Bäumen mit einer dichten Krone aus vierkantigen Ästen. Geschützt werden die Pflanzen durch ihren stark ätzenden Milchsaft und von paarweise wachsenden Dornen, neben denen im Frühsommer die gelbgrünen, oft rot getönten Blüten erscheinen. Weitere Arten aus dem südlichen und östlichen Afrika sind E. cooperi, E. grandicornis, E. avasmontana und E. grandialata. Neben diesen großen, strauch- oder baumförmigen Arten werden auch kleinere Euphorbien gezeigt. E. aggregata bildet durch Verzweigung des Hauptsprosses ein Polster von 5 bis $50 \mathrm{~cm}$ Höhe, vom Grund aus verzweigt mit acht-, bis neunkantigen, bis 3,5 cm starken,

Abb. 16 (oben): Hoch aufragende Browningia hertlingiana.

Abb. 17 (Mitte): Blüte von Stetsonia coryne.

Abb. 18 (unten): Anzucht von jungen

Euphorbia balsamifera.
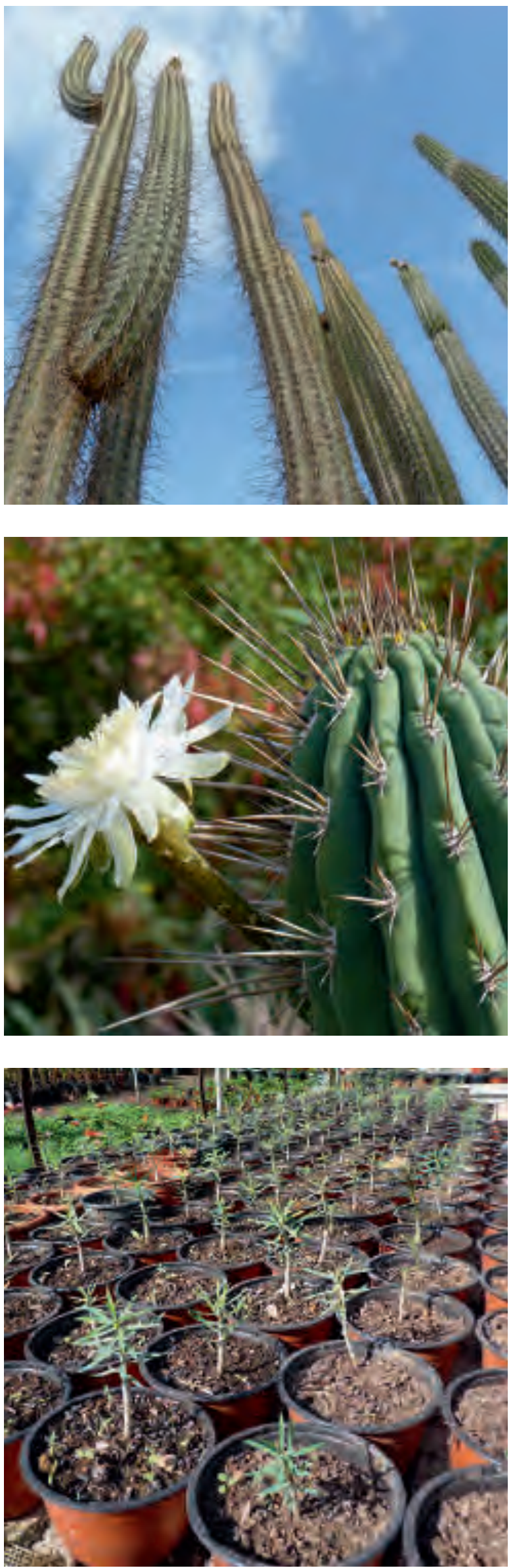


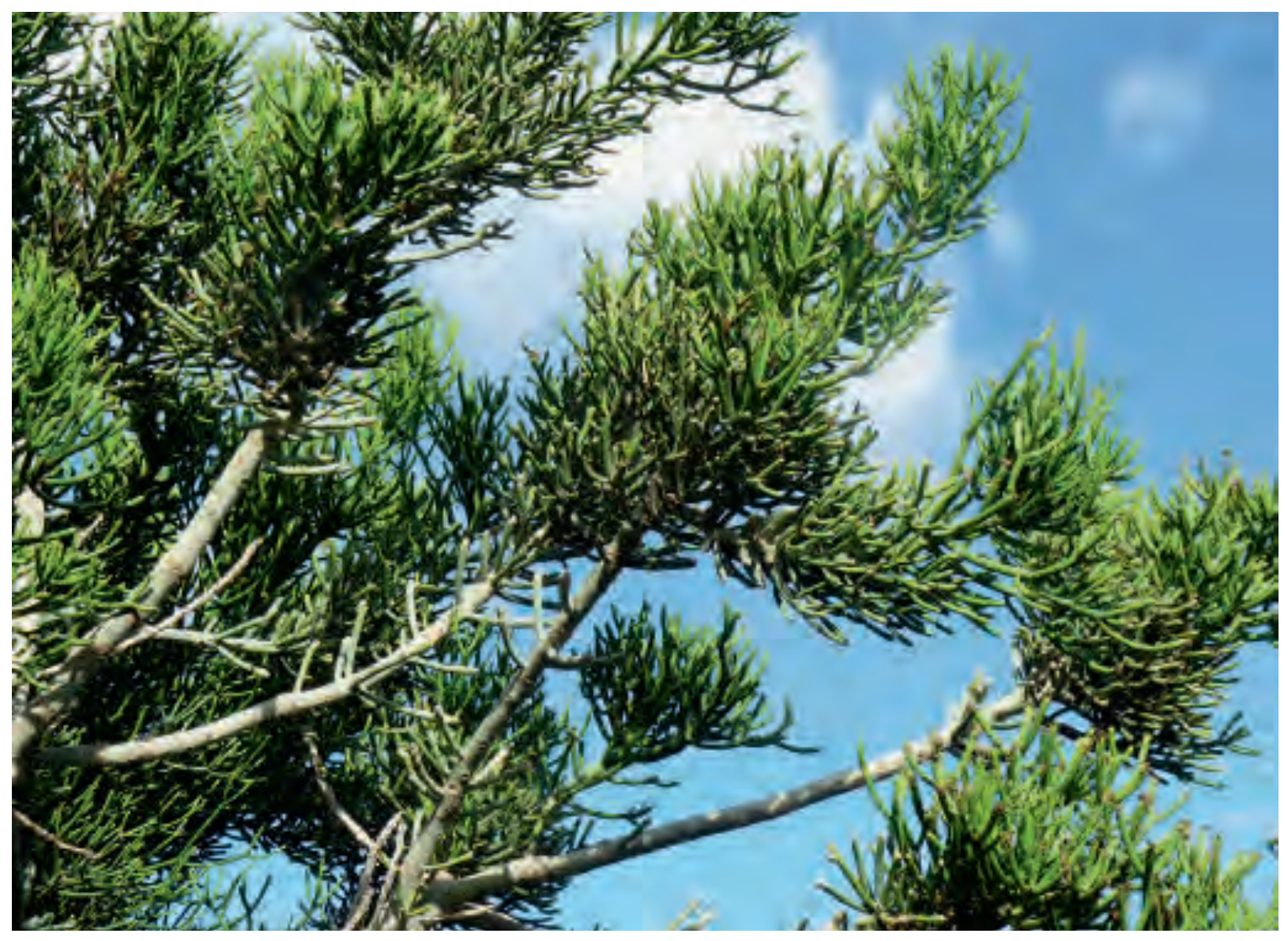

dornigen Ästen, deren Kanten durch scharfe Furchen getrennt sind. Unter den einheimischen Euphorbien ist E. handiensis hervorzuheben, ein seltener Fuerteventura-Endemit, der nur an drei Orten auf der Jandía-Halbinsel vorkommt. Zu den Wolfsmilchgewächsen gehören auch die ca. 175 Arten der Gattung Jatropha. Im Sukkulentengarten steht an mehreren Stellen Jatropha curcas, die Purgiernuss aus dem tropischen Amerika, die in vielen anderen Tropenländern eingeschleppt ist oder kultiviert wird. Sie bildet einen sukkulenten Strauch oder kleinen Baum mit verdicktem Rhizom und efeuähnlichen Blättern. Die Samen sind denen der Rizinuspflanze ähnlich, aber nicht marmoriert und ebenso wie deren Pressrückstände giftig. Das in der Pharmazie als Semen Ricini majoris bezeichnete, aus den Samen gepresste Öl wurde früher wie Rizinusöl als Abführmittel genutzt, aber auch als Brennöl verwendet. Es eignet sich zur Verarbeitung zu Biodiesel, das in speziell angepassten Motoren direkt genutzt werden kann. Auch J. podagrica, J. integerrima und J. pinnatifida sind im botanischen Garten zu sehen.

Besonders zahlreich sind im Oasis Park die Kakteen vertreten, darunter viele Säulenkakteen der Gattungen Pachycereus, Stenocereus, Stetsonia und Browningia. Manche Exemplare des Pachycereus pringlei, die jetzt ca. 35 Jahre alt sind, erreichen 5-6 m Höhe. Sehr groß wird auch $P$. weberi, $P$. pecten-aboriginum hat etwas schlankere Stämme, seine hellbraunen, einer großen Esskastanie ähnelnden Früchte sind lang bestachelt. Eine weitere Gruppe von baumförmigen Säulenkakteen wird durch Echinopsis atacamensis repräsentiert, deren zylindrische Triebe am Naturstandort bis $40 \mathrm{~cm}$ Durchmesser aufweisen können. Des Weiteren werden 12 Opuntienarten (Feigenkakteen) kultiviert. Eindrucksvoll ist Opuntia galapageia, die

Abb. 19 (oben): Euphorbia tirucalli.

Abb. 20 (Seite 61): Adenium somalense 
Galapagos-Opuntie. Sie wird in der Roten Liste gefährdeter Arten der IUCN als bedroht eingestuft. Hier im Garten wächst sie zu ansehnlichen Bäumchen heran. Die großen, vielrippigen, gelb bedornten Goldkugelkakteen (Echinocactus grusonii) stehen in ihrem Verbreitungsgebiet im zentralmexikanischen Hochland vor der Ausrottung und deshalb unter Schutz. Gleichwohl kann man den beliebten Kugelkaktus in vielen Ländern kaufen, da es genügend Gärtnereien gibt, die ihn in großen Mengen aus Samen heranziehen. Die massigen Pflanzen können einen Durchmesser von über einem Meter erreichen. Cereus jamacaru bildet hohe, derbe, graugrüne, eine Wachsschicht tragende Säulen mit kandelaberartigen Kronen, die in der Jugend aus blaugrau schimmernden Seitenästen bestehen. Der Stammdurchmesser kann bis $35 \mathrm{~cm}$ bei einer Höhe um $9 \mathrm{~m}$ betragen. Verschiedene Ferocactus-, Echinocereus, Mammillaria- und andere Kakteen-Arten vervollständigen das Bild.

Auch die aus Madagaskar stammenden Didieraceae sind im Garten vertreten. Manche Arten erreichen an ihren natürlichen Wuchsorten 8 bis $10 \mathrm{~m}$ Höhe. Didierea madagascariensis ist ein dicht bedornter, im Alter bis $40 \mathrm{~cm}$ dicker, verholzter Baum, dessen Astkrone aus schlanken, an der Spitze zuweilen übergebogenen Langtrieben besteht. In der Jugend bildet er 1 bis $2 \mathrm{~m}$ hohe, oben keulig verdickte Triebe von bis $10 \mathrm{~cm}$ Dicke. Die langen, nadelähnlichen, spiralig angeordneten Blätter bilden am Ende der Säule einen Schopf. Sie fallen während der Winterruhe ab. An ihrer Basis stehen lange, dünne Stacheln. Eine eindrucksvolle Pflanze des Gartens ist auch Adenium somalense, die flaschenförmig aufgetriebene Stämme bildet und leuchtend rosa Blüten hat.

Es ist geplant, den Bestand des botanischen Gartens des Oasis-Parks in den nächsten Jahren laufend zu erweitern. Es sollen u. a. spezielle Bereiche für Pflanzen aus den Trockengebieten Madagaskars bzw. der Insel Sokotra angelegt werden, die dem interessanten Garten zusätzlichen Reiz geben werden.

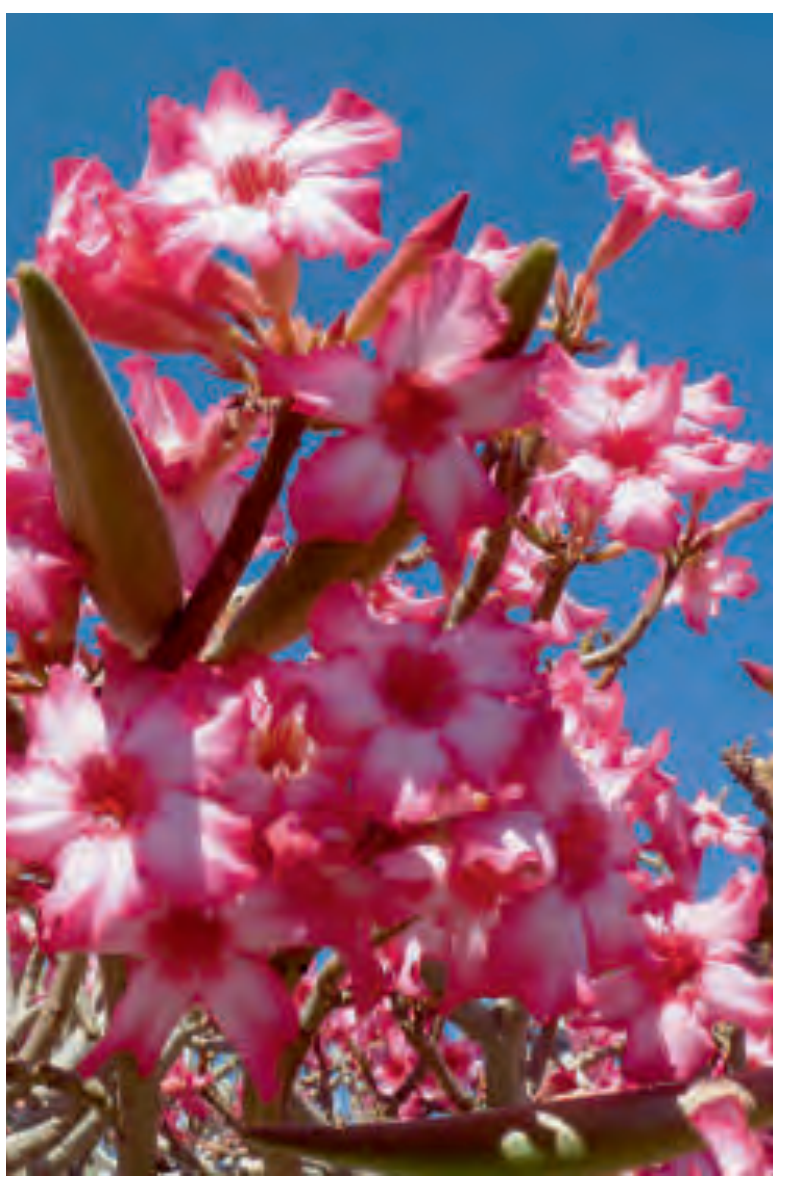

\section{Literatur}

Acebes, J. R., León, M .C., Rodríguez, M. L., del Arco, M, García, A., Pérez de Paz, P. L., Rodríguez, O., Martín V. E. \& Wildpret, W. 2oio: Pretidophyta, Spermatophyta. In: - Arechavaleta, M., Rodríguez, S., Zurita, N. \& Carcía, A. (CoOrd.) 20io: Lista de especies silvestres de Canarias. Hongos, plantas y animales terrestres 2009. - La Laguna.

Martín, V. E., Wildpret W. \& Scholz, S. 2011: Relict ecosystems of thermophilous forest as biodiversity hotspots in Fuerteventura, Canary Islands. - Plant Biosystems 145, Suppl.: $180-185$.

Reyes-Betancort, A. \& Santos, A. 2010: Gymnosporia cryptopetala Reyes-Bet. \& A. Santos (Celastraceae), a new species from the Canary Islands. - Candollea 65: 189-196. Santos, A. \& Fernández, M. 1984: Notas florísticas de las islas de Lanzarote y Fuerteventura (I. Canarias). Anal. Jard. Bot. - Madrid 41: 167-174.

SAntos, A. \& Fernández, M. 1986: Salvia herbanica spec. nova (Labiatae) en la flora de Fuerteventura (I. Canarias). - Lazaroa 9: 51-54.

Scholz, S. \& Reyes-Betancort, A. 2013: Una nueva especie del género Ferula (Apiaceae) de Fuerteventura, islas Canarias. - Vieraea 41: 173-183.

Will, M. \& Classen-Bockhoff, R. 2014: Why Africa matters: evolution of old world Salvia (Lamiaceae) in Africa. - Ann. Bot. 114: 61-83. 